\title{
Mixing Device for Generating Simple Chromatographic Gradients
}

\author{
JOINN C. IIEGENAUER, KENNETH D. TARTOF, AND \\ GEORGE W. NACE \\ From the Department of Zoology, Lniversity of Michigan, Ann Arbor
}

Received January 21, 1965

The "stand-pipe" principle has been extensively used in the design of gradient elution systems for ion-exchange chromatography (1-7). The cheapest and least complicated devices for producing simple linear, concave, or convex gradients (1-4) have utilized hydrostatic leveling between a limiting eluent reservoir (containing the final concentration desired) and a mixing chamber (initially filled with starting eluent). Eluent withdrawn from the system via the mixing chamber, which must be provided with mechanical stirring, follows a concentration gradient which is a function of the area ratio of the two vessels. Any desired simple gradient may be obtained either by selecting reservoir containers of appropriate cross section or by introducing objects of uniform cross section into the containers to adjust the area ratio.

Most of these devices employ siphons or tubing interconnections and are therefore cumbersome to position and assemble. Extensive modification of geometries is sometimes involved in producing different types of gradients, and frequently the introduction of objects into the mixing vessel obstructs efficient stirring. We describe here a general arrangement of two fixed reservoirs which can be constructed to deliver a linear gradient but is easily adapted for concave or convex gradients, or for a logarithmic gradient.

\section{Construction of Linear Gradient Device}

Reservoirs of equal area may be fashioned compactly and inexpensively from several combinations of concentrically arranged acrylic cylinders (Fig. 1). The portion of the larger cylinder not occupied by the smaller one constitutes the outer (limiting eluent) reservoir, containing the same volume as the smaller cylindrical mixing chamber at hydrostatic equilibrium. Interconnection of the reservoirs is made by a narrow U-channel (E, Fig. 1), which effectively eliminates diffusion between the 


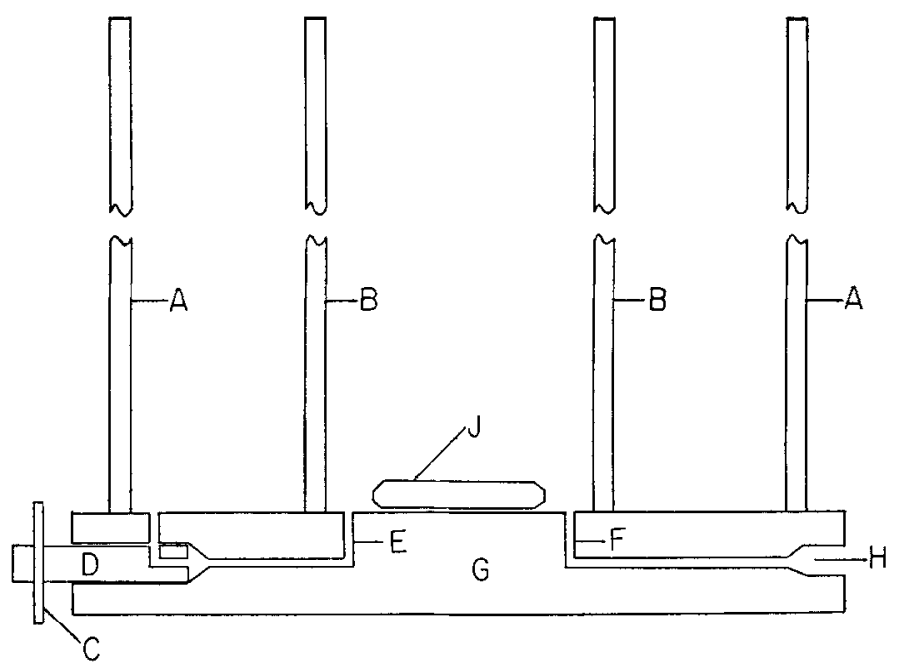

FIG. 1. Cross-sectional diagram of gradient device showing relative positions of essential ingredients. Outer and inner cylinders $A$ and $B$ are glued to $1 / 2$ " acrylic plate (G) to form fixed concentric reservoirs of equal area. Plastic rod $\mathbf{C}$ allows rotation of a snugly fitting stopcock of $1 / 4^{\prime \prime}$ Teflon rod $\mathrm{D}$, which is drilled to match configuration of the $5 \%$ " U-channel (E) connecting outer and inner reservoirs. The central mixing chamber, shown with magnetic stirring bar $\mathbf{J}$ in place, is provided with narrow outflow channel F. Connection is made to a chromatographic column with plastic tubing, which may be fit directly into undersized expansion $\mathbf{H}$.

two chambers and allows sufficiently rapid equilibration to accommodate fast chromatographic flow rates. At one portion of the channel, a "stopcock" (D, Fig. 1) is interposed to allow convenient priming and to permit independent filling of the reservoirs.

A large variety of cylinders is commercially available, and the selection of appropriate sizes to produce "matching area" combinations is tedious. Table 1 provides a convenient listing of available "matching area" cylinder combinations ${ }^{1}$ with less than $2 \%$ difference in the reservoir areas as calculated from the nominal diameters of the two cylinders.

\section{Modification for Nonlinear Gradients}

Concave or Convex Gradients. The area ratio of many of the reservoir combinations of Table 1 may be altered systematically for the production of a variety of convex and concave gradients by nesting different numbers of close-fitting acrylic cylinders around or inside the fixed

"Specifications for "Cadco" cast acrylic resin tubes, Teflon rod, and cementing materials available from: Cadillac Plastic and Chemical Co., 15111 Second Ave., Detroit 3, Michigan. Uniform inventories prevail among many manufacturers. 
TABLE 1

Suggested Combinations of Commercially Avatlable Cylinders for the Construction of Fixed "Matching Area" Reservolrs to Generate a Linear Gramifnt

$A_{i}, A_{0}=$ areas computed from inner and outer diameters of cylinders. $A_{l_{1}} A_{0}=$ areas of reservoirs (mixing chamber and limiting eluent reservoir, respectively) resulting from the combination of these cylinders. $A_{O}=A_{i}$ (outer cylinder $-A_{o}$ (inner cylinder). Diameters and areas in inches to conform to manufacturers' specifications $\left(1\right.$ in. ${ }^{2}=6.45$ $\mathrm{cm}^{2}$ ).

\begin{tabular}{|c|c|c|c|c|c|c|c|}
\hline \multicolumn{2}{|c|}{ Outer cylinder } & \multicolumn{5}{|c|}{ Inner cylinder } & \multirow{2}{*}{$\begin{array}{l}\text { Ratio of } \\
\text { reservoir } \\
\text { areas, } \\
A_{I} / A_{0}\end{array}$} \\
\hline i.d. & $A_{i}$ & o.d. & i.d. & $A_{0}$ & $A_{i}\left(A_{I}\right)$ & $A o$ & \\
\hline 1.875 & 2.761 & 1.500 & 1.125 & 1.767 & 0.994 & 0.994 & 1.000 \\
\hline 2.125 & 3.547 & 1.625 & 1.375 & 2.074 & 1.485 & 1.473 & 1.008 \\
\hline 2.500 & 4.909 & 2.000 & 1.500 & 3.142 & 1.767 & 1.767 & 1.000 \\
\hline 3.000 & 7.069 & 2.250 & 2.000 & 3.976 & 3.142 & 3.093 & 1.016 \\
\hline 3.375 & 8.946 & 2.500 & 2.250 & 4.909 & 3.976 & 4.037 & 0.985 \\
\hline 3.625 & 10.321 & 2.750 & 2.375 & 5.940 & 4.430 & 4.381 & 1.011 \\
\hline 4.000 & 12.566 & 3.000 & 2.625 & 7.069 & 5.412 & 5.497 & 0.984 \\
\hline 4.250 & 14.186 & 3.125 & 2.875 & 7.670 & 6.492 & 6.516 & 0.996 \\
\hline 4.500 & 15.904 & 3.375 & 3.000 & 8.946 & 7.069 & 6.958 & 1.016 \\
\hline 4.875 & 18.666 & 3.625 & 3.250 & 10.321 & 8.296 & 8.345 & 0.994 \\
\hline 5.125 & 20.629 & 3.750 & 3.500 & 11.045 & 9.621 & 9.584 & 1.004 \\
\hline 5.500 & 23.758 & 4.000 & 3.750 & 12.566 & 11.045 & 11.192 & 0.987 \\
\hline 5.750 & 25.967 & 4.250 & 3.875 & 14.186 & 11.793 & 11.781 & 1.001 \\
\hline 7.250 & 41.283 & 5.250 & 5.000 & 21.648 & 19.635 & 19.635 & 1.000 \\
\hline
\end{tabular}

center cylinder to reduce the area of the outer or inner reservoirs, or both. Figure 2 shows an assortment of gradients that could be produced if combinations of a series of 8 cylinders were nested into one of the linear gradient generators of Table 1 . These gradients were calculated from the general formula (1):

$$
1-C=(1-v)^{A_{0} / A_{i}}
$$

where $C=$ fraction of the limiting concentration attained when a fraction, $v$, of the total volume has been withdrawn from the mixer, and $A_{o}, A_{i}=$ areas of the outer reservoir and inner mixing chamber, respectively.

Logarithmic Gradient. When the central mixing chamber of any of the linear gradient generators of Table 1 is converted to a constant-volume reservoir by capping it tightly with a rubber' stopper, a logarithmic gradient (8) can be generated having characteristics circumscribed by the formula (1):

$$
1-C=e^{-0}
$$






Fig. 2. Assortment of gradients possible when one of the linear gradient generators selected from Table 1 is modified with different combinations of 8 nesting cylinders. The area ratio (outer reservoir/inner mixing chamber) generating each curve is indicated.

where $C=$ fraction of the limiting concentration attained when a fraction, $v$, of the fixed volume in the closed reservoir has been withdrawn from the mixer. Note that attainment of a satisfactory end point for such a gradient may require that an excess of limiting eluent in the outer reservoir be provided by refilling or initial adjustment.

\section{DISCUSSION}

The gradients generated by our device have been in excellent agreement with those calculated for different combinations of cylinders, although some deviation has been detected, probably attributable to the error inherent in some of the "matching area" combinations and the error introduced by manufacturing tolerances. These considerations do not apply if the actual area ratio is determined by measuring the volumes required to bring the solutions in the reservoirs to the same level. This method also provides direct visualization of the volume corrections that may be necessary to achieve equal hydrostatic pressures 
in the two reservoirs with eluent solutions of appreciably different densities.

The compactness, simple design, and low cost (under $\$ 25$ ) of this device recommend it for the production of many frequently required gradients.

\section{SUMMARY}

A linear gradient device consisting of two concentric reservoirs of equal cross-sectional area, in which the initial and limiting eluent solutions are maintained in hydrostatic equilibrium, may be constructed from several combinations of commercial acrylic resin cylinders. By the use of nesting cylinders, the areas of the reservoirs may be reduced independently, allowing an assortment of nonlinear gradients to be produced by simple adjustment of the area ratio of the two reservoirs.

\section{$\Lambda$ ChNNOWLEDGMENTS}

Supported in part by NIH grant CA 06929 and by an NIH grant GM 09203 to Tahir M. Rizki. We thank James E. Mite for technical assistance and John M. Allen for advice and criticisms.

\section{REFERENCES}

1. Petrrson, E. A., ANd Sober, H. A., in "Methods in Enzymology" (S. P. Colowick and N. O. Kaplan, eds.), Vol. V, p. 3. Academic Press, New York, 1962.

2. Bock, R. M., AND Ling, N.-S., Anal. Chem. 26, 1543 (1954).

3. Parr, C. W., in Proc. Biochem. Soc., 324th meeting, Biochem. J. 56, xxvii (1954).

4. KATZ, S., Anal. Biochem. 5, 7 (1963).

5. Peterson, E. A., and Sober, H. A., Anal. Chem. 31, 857 (1959).

6. Peterson, E. A., ANd Rowland, J., J. Chromatog. 5, 330 (1961).

7. Anderson, N. G., Bond, H. E., and Cannina, R. E., Anal. Biochem. 3, 472 (1962).

8. Lakshmanan, T. K., and Leeberman, S., Arch. Biochem. Biophys. 45, 235 (1953). 